\title{
1. Distinction Between Active and Passive Targeting of Nanoparticles Dictate Their Overall Therapeutic Efficacy
}

\author{
${ }_{3}$ Tristan D. Clemons, ${ }^{* \dagger,}{ }^{\dagger} \perp$ Ruhani Singh, ${ }^{\dagger, \dagger, \perp}$ Anabel Sorolla, ${ }^{\S}$ Nutan Chaudhari, ${ }^{\dagger}$ Alysia Hubbard, ${ }^{\|}$ \\ 4 and K. Swaminatha Iyer ${ }^{\dagger, *(0)}$ \\ $5{ }^{\dagger}$ School of Molecular Sciences, University of Western Australia, 35 Stirling Highway, Crawley, Western Australia Australia, 6009 \\ $6{ }^{*}$ CSIRO Manufacturing, New Horizons Centre, 20 Research Way, Clayton, Victoria Australia 3168 \\ $7{ }^{\S}$ Harry Perkins Institute of Medical Research, 6 Verdun Street, Nedlands, Western Australia Australia 6009 \\ ${ }_{8}$ "Centre for Microscopy, Characterisation and Analysis, University of Western Australia, 35 Stirling Highway, Crawley, Western \\ 9 Australia Australia, 6009
}

11 ABSTRACT: The role of nanoparticles in cancer medicine is 12 vast with debate still surrounding the distinction between 13 therapeutic efficacy of actively targeted nanoparticles versus 14 passively targeted systems for drug delivery. While it is 15 commonly accepted that methodologies that result in homing 16 a high concentration of drug loaded nanoparticles to the 17 tumor is beneficial, the role of intracellular trafficking of these 18 nanoparticles in dictating the overall therapeutic outcome 19 remains unresolved. Herein we demonstrate that the 20 therapeutic outcome of drug loaded nanoparticles is governed 21 beyond simply enabling nanoparticle internalization in cells.

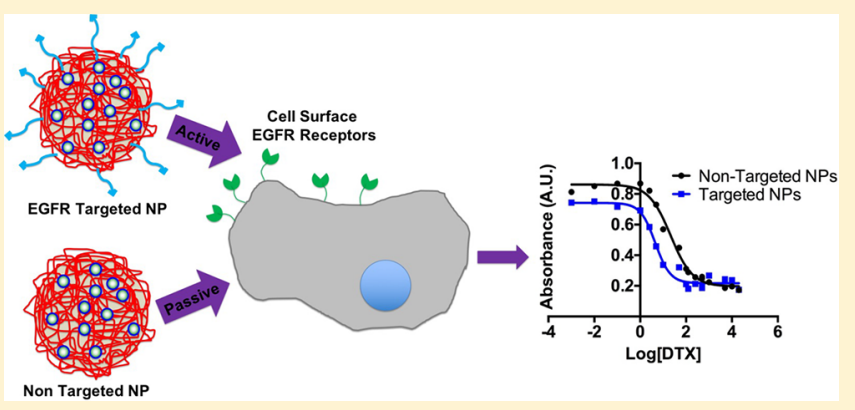
Using two model polymeric nanoparticles, one decorated with the GE11 peptide for active targeting of the epidermal growth factor receptor (EGFR) and the other without, we demonstrate that EGFR mediated intracellular internalization results in an enhanced therapeutic effect compared to the nontargeted formulation. Our findings demonstrate that the intracellular destination of nanoparticles beyond its ability to internalize is an important parameter that has to be accounted for in the design of targeted drug delivery systems.

28 Despite significant advances in cancer treatments and 29 diagnosis, cancer still remains as one of the world's most 30 devastating diseases with more than 10 million new cases 31 reported every year. ${ }^{1}$ Concomitant with these devastating 32 cancer incidences are the side effects of chemotherapeutics still 33 widely used for the treatment of this disease. Chemo34 therapeutics are the major class of drugs available in the 35 arsenal against cancer, introduced in the 1950s for the 36 treatment of these diseases. ${ }^{2}$ However, these drugs are highly 37 toxic and can result in severe and debilitating side effects for 38 patients. ${ }^{3,4}$ As a result of this, chemotherapeutics encapsulated 39 in nanoparticle delivery vehicles are a promising field to 40 enhance the effectiveness of these treatments in cancer while 41 avoiding some of these side effects. ${ }^{5-8}$ There is great interest in 42 applying nanoparticle technology to cancer therapies largely 43 resulting from the appealing features that nanoparticles 44 possess. These include therapeutic protection from degrada45 tion, improved drug pharmacokinetics, improved intracellular 46 penetration, selective tissue targeting, and the inclusion of 47 imaging modalities. ${ }^{9-11}$ Further to this, the increasing 48 opportunities of "theranostic" nanoparticles which can 49 combine both diagnostic capabilities as well as therapy within a single entity provide great promise in the fight against 50 cancer. $^{12,13}$

Nanoparticles due to their unique size are able to exploit the 52 distinct cancer pathology and its molecular biology to in turn 53 result in higher uptake and preferential targeting of 54 therapeutics to the tumor compared to traditional treatments. ${ }^{8} 55$ Broadly, this is achieved by two methods, "passive" or "active" 56 targeting. Passive targeting is possible due to the unique 57 changes in the cancer vasculature. Due to the rapid growth of 58 tumors, blood vessels and junctions are not formed properly 59 and can be loose and leaky. Owing to the unique size of 60 nanoparticle formulations, they are able to pass through these 61 loose junctions resulting in preferential accumulation at the 62 tumor site over time. This phenomenon is known as the 63 enhanced permeation and retention (EPR) effect. ${ }^{10,14}$ As a 64 result of this and the combinatorial advantages of nanoparticle 65 based delivery vehicles, a number of systems have been 66 approved by the U.S. Food and Drug Administration (FDA) 67 for chemotherapeutic delivery. These formulations include 68

Received: August 29, 2018

Revised: November 1, 2018

Published: November 15, 2018 
69 Doxil (approved 1995), a liposomal delivery system of 70 doxorubicin, Abraxane (approved 2005), an albumin based 71 nanoparticle delivery system of paclitaxel, and in Korea a 72 polymeric micelle delivery vehicle of paclitaxel, Genexol-PM 73 (approved in Korea 2007), have recently been approved. ${ }^{15}$ All 74 of these delivery systems are passively targeted formulations 75 and they can suffer from a number of limitations. Lack of 76 control in uptake in these systems can result in off target drug 77 delivery, which may lead to multiple drug resistance, a situation 78 where chemotherapy treatments fail in patients due to the 79 resistance of cancer cells to one or more drugs. ${ }^{10,16}$ Another 80 limitation of the passive strategy is that certain tumors do not 81 strongly exhibit the EPR effect, and the permeability of vessels 82 can be highly heterogeneous throughout a single tumor. ${ }^{16}$

83 Active targeting of nanoparticles is where a targeting moiety 84 (e.g., ligand, antibody, peptide) is introduced onto the 85 nanoparticle system to target specific changes in cancer cell 86 biology, which are highly upregulated in comparison to the 87 healthy surrounding cells and tissues. ${ }^{10,17}$ In this process, 88 nanoparticles will recognize and bind to target cells through 89 ligand-receptor interactions, and the bound nanoparticles are 90 internalized before the drug is released inside the cell, resulting 91 in less off target drug release compared to passively targeted 92 systems. One method to improve the retention of nano93 particles and in turn their therapeutic payload in cancer tumors 94 is active targeting to cell membrane receptors that are 95 overexpressed in cancer cell lines, such as the epidermal 96 growth factor receptor (EGFR). ${ }^{18,19}$ EGFR up regulation has 97 been implicated in the aggressiveness of several cancers 98 including breast (overexpression evident in approximately $9950 \%$ of triple negative breast cancer tumors), ${ }^{20}$ renal, ovarian, 100 colon, and nonsmall cell lung cancer. ${ }^{21}$ As a result of this 101 targeting, the EGF receptor is an exciting prospect for actively 102 targeted chemotherapeutic nanoparticle formulations in the 103 treatment of a range of cancers.

104 It is now widely accepted that irrespective of the targeting 105 strategy adopted in the design for drug delivery, i.e., active or 106 passive, it is pivotal for the nanoparticles to become 107 therapeutically relevant, and their intracellular uptake is a 108 crucial factor. Furthermore, it is widely established that the 109 cellular internalization is highly dependent on the size, shape, 110 and surface chemistry of the nanoparticles. However, what 111 remains poorly understood is how the intracellular trafficking 112 and intracellular destination of nanoparticles affect the overall 113 therapeutic outcome. In this study, we show that intracellular 114 destination of an actively targeted polymeric nanoparticle and a 115 passively targeted nanoparticle with comparable internalization 116 abilities differ vastly in their overall therapeutic outcome. We 117 demonstrate this using docetaxel (DTX) loaded polymeric 118 nanoparticles with and without GE11 peptide for targeting the 119 EGFR, which is highly overexpressed in a number of cancers. ${ }^{21}$

\section{RESULTS AND DISCUSSION}

121 The role of nanoparticle targeting is of great interest in the 122 application of nanoparticles for the treatment of disease and 123 injuries, especially their role in cancer medicine. Despite this 124 interest, there are limited studies available which look to 125 compare actively targeted systems, such as the ERP effect, with 126 passively targeted systems. This study demonstrates the 127 superior effectiveness in relevant in vitro cancer models of an 128 EGFR targeted polymeric nanoparticle delivery vehicle of 129 docetaxel (DTX) to a nontargeted nanoparticle comparison. 130 The polymeric nanoparticles were prepared by an oil-in-water emulsion process where poly(glycidyl methacrylate) (PGMA) 131 was first synthesized and then modified with 6-maleimidohex- 132 anoic acid and rhodamine B through epoxide ring opening 133 reactions. The attachment of the rhodamine B fluorescent 134 probe allowed for in vitro fluorescent tracking of the 135 nanoparticles, while the 6-maleimidohexanoic acid modifica- 136 tion was used to anchor the GE11 EGFR targeting peptide to 137 the surface of the nanoparticle. The GE11 peptide, with 138 sequence YHWYGYTPQNVI as published previously, ${ }^{22,23}$ was 139 synthesized with a custom tail of four glycine units and a 140 cysteine residue (YHWYGYTPQNVIGGGGC) to allow for 141 consistent and precise attachment to the nanoparticle surface 142 through thiol-maleimide chemistry. The four-glycine linkers 143 providing flexibility in the GE11 targeting moiety away from 144 the nanoparticle surface an important consideration for 145 targeting peptides. $^{24,25}$ For DTX-loaded nanoparticles, the 146 chemotherapeutic was dissolved in the "oil" phase during the 147 emulsion process for encapsulation.

The nanoparticles are approximately $190 \mathrm{~nm}$ in diameter 149 (PGMA-DTX-GE11, 195.3 nm, PDI 0.104; PGMA-DTX, 150 $189.3 \mathrm{~nm}$, PDI 0.102) with the GE11 targeted nanoparticles 151 exhibiting a higher zeta potential (PGMA-DTX-GE11, 24.2 \pm 152 $5.8 \mathrm{mV}$; PGMA-DTX NPs, $-12.6 \pm 8.4 \mathrm{mV}$ ) (Figure 1) $153 \mathrm{fl}$

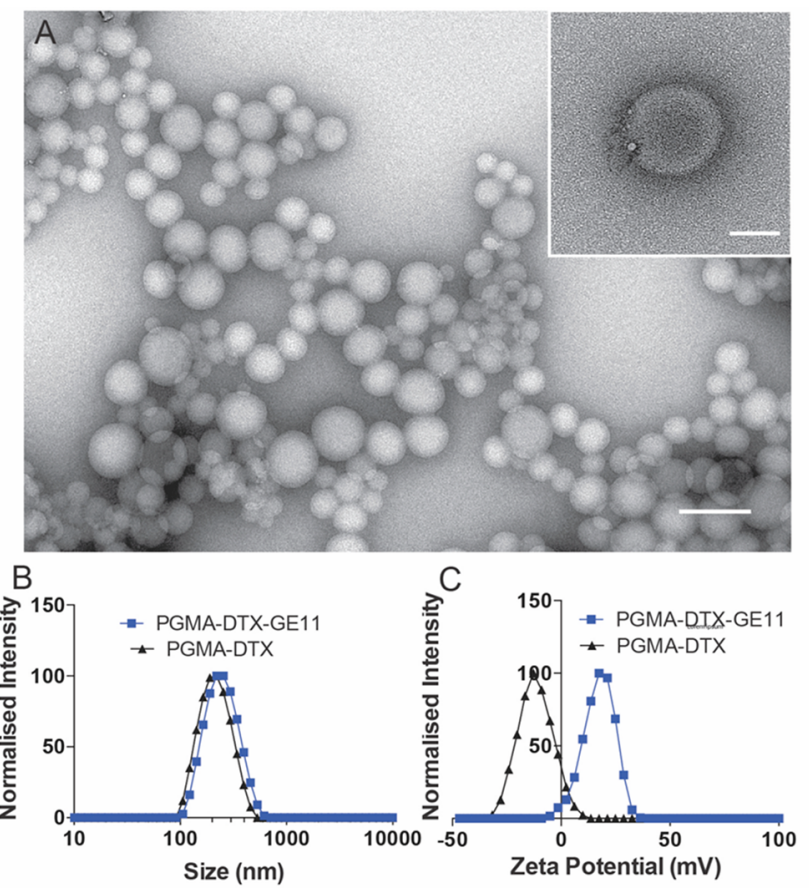

Figure 1. Physical characterization of the targeted DTX loaded nanoparticles. (A) Transmission electron microscopy images of the GE11 targeted DTX loaded nanoparticles with high magnification inset (scale $200 \mathrm{~nm}$, inset $50 \mathrm{~nm}$ ), (B) dynamic light scattering assessment of nanoparticle size, and (C) zeta potential for the targeted and nontargeted nanoparticle formulations.

resulting from the attachment of the slightly cationic peptide. 154 Drug loading was assessed by reverse phase high-pressure 155 liquid chromatography (HPLC) with the GE11 targeted 156 nanoparticles found to contain $24.0 \pm 1.3 \% \mathrm{w} / \mathrm{w}$ DTX, 157 whereas the nontargeted nanoparticles contained a load of $21.8 \quad 158$ $\pm 0.8 \% \mathrm{w} / \mathrm{w}$ (see the Supporting Information Figure S1 for the 159 standard curve). The slight difference between particle loading 160 is a result of batch-to-batch variation in the nanoparticle 161 

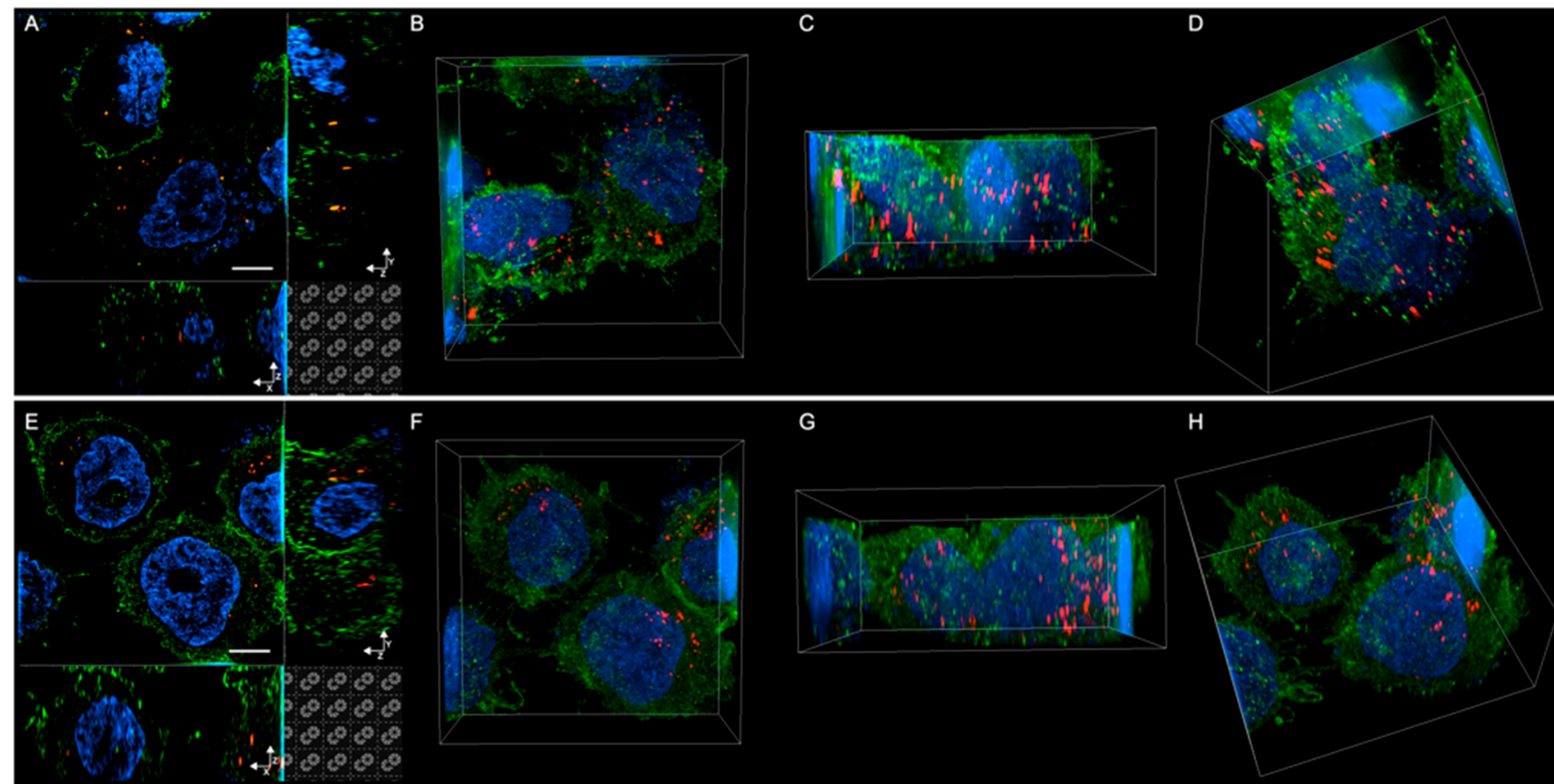

G

H

Figure 2. High-resolution confocal microscopy and structured illumination microscopy (SIM) assessment of nanoparticle internalization. (A-D) GE11-PGMA targeted nanoparticles and (E-H) PGMA nontargeted nanoparticles after $24 \mathrm{~h}$ incubation with MDA-MB-231 cells. (A) Highresolution confocal microscopy image taken from a central slice of the z-stack showing GE11-PGMA targeted internalization with the $x-y$, $z-y$, and $z-x$ profiles displayed. 3D reconstructions of the stack obtained from SIM images (B) top, (C) side, and (D) isometric views. (E) Highresolution confocal microscopy image taken from a central slice of the $z$-stack showing PGMA nontargeted internalization with the $x-y, z-y$, and $z-x$ profiles displayed. 3D reconstructions of the stack obtained from SIM images (F) top, $(\mathrm{G})$ side, and $(\mathrm{H})$ isometric views. Hoescht labeling of the nuclei (blue), anti-EGFR (green), and rhodamine labeled nanoparticles (red). Scale bars are $5 \mu \mathrm{m}$.

162 emulsion preparation process. Quantification of the maleimide 163 functionalization and the subsequent attachment of GE11 164 targeting peptide was achieved using a Fluorometric Thiol 165 Quantification Assay Kit. Attachment of GE11 peptide was 166 found to be $22.4 \mathrm{nmol} \mathrm{mg} \mathrm{mg}^{-1}$ of the targeted nanoparticle 167 formulation (see the Supporting Information Figure S2). From 168 physical characterization it was evident that the nanoparticles 169 were similar in size, shape, and drug loading with the major 170 difference being the attachment of the GE11 targeting peptide. 171 Drug release was assessed by HPLC, and it was evident that 172 DTX was released at a faster rate from the PGMA nontargeted 173 nanoparticle (71.2\% DTX released) when compared to the 174 GE11 targeted nanoparticle (57.1\% released) over a period of 1756 days (Supporting Information Figure S3). At $24 \mathrm{~h}$, the 176 nontargeted formulation had released $7.2 \%$ more DTX then 177 the GE11 targeted formulation, an important result with 178 downstream in vitro experiments based on $24 \mathrm{~h}$ incubation 179 with the therapeutic nanoparticles for comparison.

180 The uptake of both the GE11 targeted and the nontargeted 181 nanoparticle without DTX loading was assessed by confocal 182 microscopy in the breast cancer cell line MDA-MB-231. MDA183 MB-231 cells were chosen as DTX has been used extensively in 184 the treatment of breast cancers, and the overexpression of 185 EGFR with these cells has been demonstrated previously. ${ }^{26,27}$ 186 It was anticipated the targeted nanoparticle would have 187 enhanced cellular association and in turn increased uptake 188 compared to the nontargeted formulation; however, this was 189 not evident by confocal microscopy (see the Supporting 190 Information Figure S4). In fact, the nontargeted nanoparticles 191 had significant cellular association most likely due to 192 nonspecific binding and cellular membrane association events. 193 To further probe nanoparticle internalization, high-resolution structured illumination microscopy (SIM) was used to confirm 194 uptake and make comparisons between the targeted and 195 nontargeted nanoparticles (Figure 2 and Supporting Informa- 196 f2 tion S5 video links of SIM 3D reconstructions of the PGMA- 197 GE11 NP and PGMA NP internalization). Cellular internal- 198 ization was clearly evident for both nanoparticles by SIM 199 microscopy, confirming both the targeted and nontargeted 200 nanoparticles were successful at internalizing within a $24 \mathrm{~h} 201$ time period.

Further assessment of the nanoparticle fate once internalized 203 was conducted by investigating the colocalization of nano- 204 particles with both endosomes and lysosomes by immunohis- 205 tochemistry (see the Supporting Information Figure S6A,B). 206 Quantitative analysis of these confocal images by comparing 207 the Pearson's correlation coefficients (a measure to compare 208 the colocalization) of both the GE11 targeted and the 209 nontargeted PGMA nanoparticles with endosomes or 210 lysosomes found significant differences in the intracellular 211 localization of the nanoparticles following $24 \mathrm{~h}$ incubation (see 212 the Supporting Information Figure S6C). Manders et al. were 213 the first to use the Pearson correlation coefficient for 214 fluorescent microscopy colocalization studies. ${ }^{28}$ Briefly, the 215 Pearson's correlation coefficient measures the pixel by pixel 216 covariance in the signal levels of two images, with a perfect 217 correlation of 1 and a perfectly inverse correlation of $-1 .^{29}$ For 218 this analysis, only the signal from the respective nanoparticle 219 images with the endosome or lysosome immunohistochemistry 220 images were compared to avoid background effects from 221 alternate fluorescent labels present in the stacked image. This 222 analysis showed the EGFR targeted nanoparticle associated 223 significantly less with endosomes and lysosomes when 224 compared to the nontargeted PGMA nanoparticle. Further to 225 
226 this, both nanoparticles associated more with lysosomes 227 compared to endosomes after $24 \mathrm{~h}$ of incubation (see the 228 Supporting Information Figure S6). Hence despite similar 229 levels of cellular internalization of the nanoparticles within 230 MDA-MB-231 cells, it is apparent that the internal trafficking 231 of these nanoparticles is affected by the EGFR targeting, which 232 may also contribute to differences in the therapeutic delivery 233 and efficacy of these nanoparticle drug delivery vehicles.

234 To assess the therapeutic efficacy of these DTX loaded 235 nanoparticles, the half maximal inhibitory concentration 236 (IC50) was determined in both MDA-MB-231 and the 237 MCF-7 breast cancer cell lines, respectively (Figure 3). In
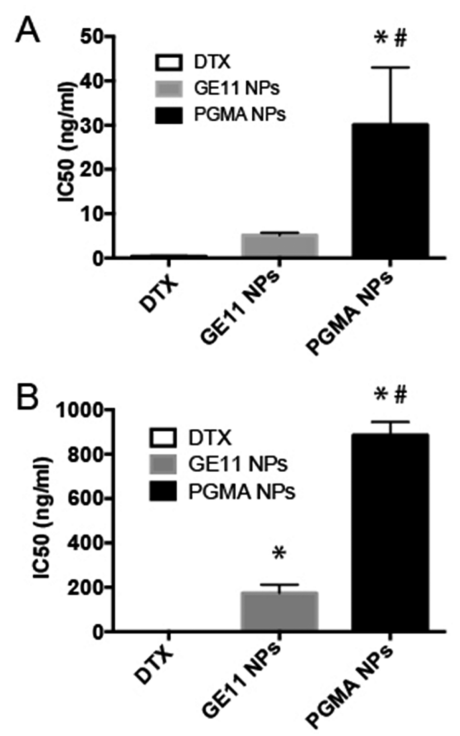

Figure 3. IC50 analysis of docetaxel (DTX), the GE11 targeted DTX nanoparticle formulation (GE11 NPs), and the untargeted PGMA DTX nanoparticles (PGMA NPs) in both MDA-MB-231 and MCF-7 cells. (A) MDA-MB-231 and (B) MCF-7 breast cancer cell lines. Data displayed as mean $\pm \mathrm{SD}$ and statistically assessed with a one-way ANOVA followed by a Bonferroni multiple-comparisons test. Significance $(* p<0.05)$ for comparison to free DTX and (\#p< 0.05) for significance between the targeted GE11 NPs and the untargeted PGMA NPs.

238 assessing the IC50 of these formulations, it was evident that 239 the GE11-PGMA targeted nanoparticles had a significantly 240 lower IC50 value compared to the untargeted formulations in 241 both MDA-MB-231 (5.1 ng/mL for GE11-PGMA NPs vs 30.1 $242 \mathrm{ng} / \mathrm{mL}$ for PGMA NPs, Figure 3A) and MCF-7 (173.5 ng/mL 243 for GE11-PGMA NPs vs $886.2 \mathrm{ng} / \mathrm{mL}$ for PGMA NPs, Figure $244 \mathrm{BB})$ cell lines. This is despite the targeted nanoparticle 245 releasing 7\% less DTX than the nontargeted nanoparticle in a $24624 \mathrm{~h}$ period. These data presented together suggest that the 247 active targeting of nanoparticles to cancerous cells results in 248 active uptake and in turn direct delivery of the chemo249 therapeutic at the site of cell activation.

250 To investigate this further and to test whether the GE11251 PGMA targeted nanoparticle was indeed acting through an 252 EGFR mediated pathway, an experiment was performed to first 253 block the EGFR receptor in culture with a blocking antibody 254 before incubation with the targeted and untargeted DTX 255 containing nanoparticles at their half inhibitory (IC50) 256 concentrations, respectively (Figure 4 ). The presence of the 257 EGFR blocking antibody by itself in culture media did not 258 significantly alter cell viability (Figure 4). Pretreatment of cells

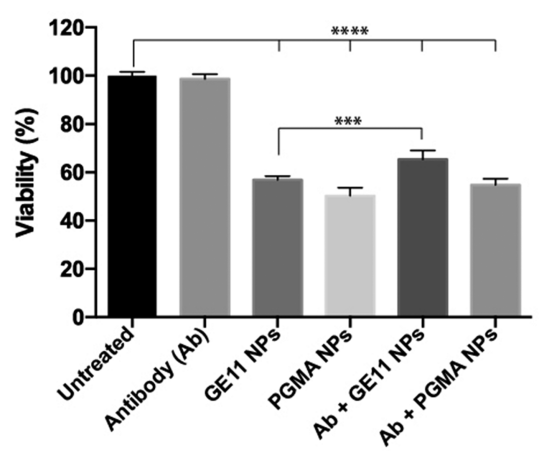

Figure 4. Blocking of the EGFR receptor significantly affects the efficacy of the GE11 targeted nanoparticle (GE11 NPs). MDA-MB231 cells were plated and preincubated with an EGFR blocking antibody for $1 \mathrm{~h}$ before treatment with the IC50 concentration of each nanoparticle formulation and compared. Data displayed as mean \pm SD and statistically assessed with a one-way ANOVA followed by a Bonferroni multiple-comparisons test. Significance $(* * * p<0.001$, $* * * * P<0.0001)$.

with this blocking antibody prior to nanoparticle treatment 259 resulted in significant rescue of cell viability compared to 260 GE11-PGMA targeted nanoparticle treatment only, but this 261 rescue was not observed for the nontargeted PGMA 262 nanoparticle (Figure 4). These data taken together support 263 an active uptake of the GE11 targeted nanoparticles mediated 264 by the EGFR due to the significant rescue observed. 265 Furthermore, the nontargeted nanoparticles are internalized 266 by cells via passive processes not dependent on the EGFR. 267 Previous studies investigating similar nanoparticles found that 268 inhibition of clathrin mediated endocytosis (chlorpromazine), 269 caveolin mediated endocytosis (nystatin/progesterone), and 270 micropinocytosis ( $N, N$-dimethylamiloride) had no significant 271 effect on PGMA based polymeric nanoparticles of similar size, 272 and hence it was assessed that these specific cellular uptake 273 pathways were not required for polymeric nanoparticle cellular 274 internalization. $^{30}$ The activation of the EGFR resulting in 275 cellular internalization via clathrin dependent uptake is also 276 supported extensively in the literature. ${ }^{31,32}$ It is evident that 277 cellular uptake of a therapeutic payload into a target cell is not 278 alone sufficient for efficacy, but the pathway of internalization 279 plays an integral role in the therapeutic outcome of 280 nanoparticle delivery agents.

\section{CONCLUSIONS}

281

Despite significant efforts, the role of active targeting moieties 283 in nanomedicine versus passively targeted systems still remains 284 unclear. In this study, we present data on two model polymeric 285 drug delivery agents: one actively targeted through decoration 286 of the nanoparticle surface with the EGFR targeting GE11 287 peptide; and one without, enabling passive internalization by 288 cells. This study demonstrates the importance that active 289 targeting of the nanoparticle system has on dictating the 290 intracellular trafficking and furthermore the direct role that this 291 intracellular trafficking has on the efficacy of therapeutic 292 nanoparticles in cancer treatment. These results suggest that 293 careful consideration of actively targeted nanoparticles cannot 294 only influence the cellular uptake of the therapeutic cargo but 295 also have downstream ramifications on the cellular recognition 296 and intracellular processing of the cargo once inside the cell. 297 For nanoparticle drug delivery systems to reach the potential 298 they clearly possess in the treatment of cancer, thorough 299 
300 studies on the efficiency of targeting need to occur to ensure 301 optimal efficacy from these systems can be achieved.

\section{MATERIALS AND METHODS}

303 Materials. Glycidyl methacrylate (GMA, 97\%, Sigma), 2,2'304 azobis(2-methylpropionitrile) (AIBN, $0.2 \mathrm{M}$ in toluene, Sigma), 305 methyl ethyl ketone (MEK, 2-butanone; Fischer Chemical, LR grade), 306 Pluronic F-108 $\left(\mathrm{Mn}=14600 \mathrm{~g} \mathrm{~mol}^{-1}\right.$, Sigma), 6-maleimidohexanoic 307 acid (Sigma), rhodamine B (Kodak), sodium acetate (Sigma), Tris(2308 carboxyethyl)phosphine hydrochloride (Sigma), and docetaxel (LC 309 Laboratories, 97\%) were all used as received. The GE11 peptide was 310 synthesized with a GGGC linker sequence at the carboxyl terminus 311 with the following sequence (YHWYGYTPQNVIGGGGC, China 312 Peptides) and it was used as received. Tissue culture and 313 immunohistochemistry reagents such as minimum essential medium 314 (Invitrogen), fetal bovine serum (Invitrogen), Trypsin/EDTA 315 (Sigma), GlutaMAX (Invitrogen), paraformaldehyde (Sigma), 316 mouse anti-EGFR Monoclonal antibody (H11, Life Technologies, 317 catalog no. MA5-13070), Hoechst (Sigma), goat antimouse Alexa 318 Fluor 488 (Life Technologies, catalog no. A11001), EEA1 antibody 319 (Abcam, ab2900), LAMP2 antibody (Santa Cruz, sc-18822 (H4B4)), 320 goat antirabbit AF488 (ThermoFisher, A-11034), goat antimouse 321 AF647 (ThermoFisher, A-21235), and fluorobrite (Invitrogen) were 322 used as received. Coverglass used for SIM imaging were $170 \pm 5 \mu \mathrm{m}$ 323 from Marienfeld, Germany. All other reagents were of analytical 324 grade.

325 Poly(glycidyl methacrylate) (PGMA) Synthesis. PGMA (Mn = $326186200 \mathrm{~g} / \mathrm{mol}$, PDI 1.644) was synthesized by the free radical 327 polymerization of glycidyl methacrylate in methyl ethyl ketone (1:1 328 ratio) at $80{ }^{\circ} \mathrm{C}$ for $18 \mathrm{~h}$. The obtained polymer was purified by 329 multiple precipitations from the MEK solution using methanol. 330 Azobis(isobutyronitrile) (AIBN) was used as the initiator with 331 product and molecular weight confirmed by NMR and GPC, 332 respectively.

333 Synthesis of PGMA Nanoparticles. PGMA (200 mg) was 334 reacted with 6-maleimidohexanoic acid (MHA, $300 \mathrm{mg}$ ) in MEK (30 $335 \mathrm{~mL}, 70{ }^{\circ} \mathrm{C}, 5 \mathrm{~h}$ ). The solvent was removed by a rotovap to 336 approximately $2 \mathrm{~mL}$, and the MHA modified PGMA (PGMA-MHA) 337 precipitated in diethyl ether. The MHA-PGMA was reacted with 338 rhodamine $\mathrm{B}(\mathrm{RhB}, 20 \mathrm{mg})$ in $\operatorname{MEK}\left(30 \mathrm{~mL}, 70{ }^{\circ} \mathrm{C}, 5 \mathrm{~h}\right)$. The 339 PGMA-RhB-MHA was collected by precipitating in diethyl ether and 340 dried under a flow of $\mathrm{N}_{2}$ to remove residual ether.

341 PGMA nanoparticles were prepared using a nonspontaneous 342 emulsification process as described previously. ${ }^{30,33}$ The modified 343 polymer (PGMA-RhB-MHA for GE11 attachment post nanoparticle 344 formulation or PGMA-RhB for nontargeted nanoparticles without 345 GE11 modification, approximately $100 \mathrm{mg}$ ) was dissolved in a mixture 346 of chloroform $(1.5 \mathrm{~mL})$ and MEK $(4.5 \mathrm{~mL})$ along with docetaxel $(20$ $347 \mathrm{mg}$ ). This organic phase was added dropwise, with rapid stirring, to an 348 aqueous phase of Pluronic F-108 (12.5 mg mL $\mathrm{m}^{-1}, 30 \mathrm{~mL}$ ), and the 349 emulsion was homogenized with a probe-type ultrasonicator on low 350 power for $1 \mathrm{~min}$. The organics were evaporated, and the sample 351 centrifuged at $3000 \mathrm{~g}(45 \mathrm{~min})$ to remove large aggregates and excess 352 polymer. The supernatants were collected, and the nanoparticles 353 centrifuged at $20000 \mathrm{~g}(20 \mathrm{~min})$ to collect the nanoparticles. 354 Nanoparticles were washed a further 3 times with aqueous pluronic 355 F-108 $\left(12.5 \mathrm{mg} \mathrm{mL}^{-1}, 30 \mathrm{~mL}\right)$, before storage at $4{ }^{\circ} \mathrm{C}$ of the stock and 356 aliquots collected and freeze-dried for concentration determination.

357 GE11 Peptide Attachment. The GE 11 peptide was dissolved in 358 a degassed sodium acetate buffer $(50 \mathrm{mM}, \mathrm{pH} 4.5)$ prepared in 359 Pluronic F108 $\left(12.5 \mathrm{mg} \mathrm{mL}^{-1}\right)$ to a final GE11 concentration of $5 \mathrm{mg}$ $360 \mathrm{~mL}^{-1}$. 6-Maleimidohexanoic acid coated nanoparticles $(4 \mathrm{mg})$ were 361 centrifuged $(16000 \mathrm{~g}, 0.5 \mathrm{~h})$ and suspended in the acetate buffer $(50$ $362 \mathrm{mM}, 1 \mathrm{~mL}$ ). This process was repeated a second time (solution A). 363 Tris(2-carboxyethyl)phosphine hydrochloride (TCEP, $40 \mathrm{mg}$ ) was 364 dissolved in the acetate buffer $(50 \mathrm{mM}, 920 \mu \mathrm{L})$, and an aliquot of the 365 GE11 peptide stock was added $(80 \mu \mathrm{L})$ and left to incubate $(0.5 \mathrm{~h})$ at 366 room temperature (RT, solution B). Solution A was combined with 367 solution B and left to stir slowly ( $24 \mathrm{~h}, \mathrm{RT}$, under argon). Following peptide attachment, the nanoparticles were washed by repeated 368 centrifugation $(2 \times$ acetate buffer, final wash Pluronic F108 solution, 369 $16000 \mathrm{~g}, 0.5 \mathrm{~h}$ ) before suspending in $1 \times$ phosphate buffered saline 370 (PBS) for ongoing in vitro studies.

Nanoparticle Characterization. Dilute samples of nanoparticles 372 in MQ water were assessed for hydrodynamic size and zeta potential 373 by dynamic light scattering on a Zetasizer Nano ZS (Malvern 374 Instruments). Nanoparticles were drop-casted on carbon coated 375 transmission electron microscopy (TEM) grids and imaged with an 376 accelerating voltage of $120 \mathrm{kV}$ on a JEOL 2100 TEM for physical 377 characterization. Lyophilized aliquots of the nanoparticles were used 378 for drug loading determination by reverse phase high-pressure liquid 379 chromatography (RP-HPLC). The lyophilized aliquots were weighed 380 before being suspended in methanol $(1 \mathrm{~mL})$. The suspension was 381 sonicated and vortexed to dissolve the encapsulated docetaxel. The 382 nanoparticles were removed via centrifugation $(16000 \mathrm{~g}, 0.5 \mathrm{~h})$, and 383 the supernatant containing the dissolved free drug was analyzed using 384 RP-HPLC. The measurements were performed on a Waters 2695385 separation module with a Waters $2489 \mathrm{UV} /$ vis detector (determi- 386 nation $\lambda=232 \mathrm{~nm}$ ) using reverse phase isocratic elution (methanol- 387

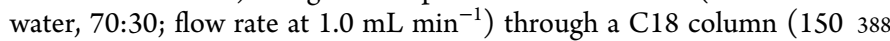
$\left.\mathrm{mm} \times 4.60 \mathrm{~mm}, 5 \mu \mathrm{m}, 25 \pm 5{ }^{\circ} \mathrm{C}\right)$. The measurements were 389 compared against a standard curve performed in identical run 390 conditions (see the Supporting Information Figure S1 for standard 391 curve). 6-Maleimidohexanoic acid and the final peptide concentration 392 attached to the nanoparticles was quantified using a fluorometric thiol 393 quantification assay kit as per the manufacturer's instructions (abcam, 394 ab112158, see the Supporting Information Figure S2 for the standard 395 curve).

396

Drug Release Experiment. Docetaxel release was assessed by 397 RP-HPLC following a previously published protocol by Singh et al. 398 using the above-mentioned running conditions for drug loading 399 characterization. ${ }^{34}$

Cell Culture. MDA-MB-231 or MCF-7 cells were cultured in 401 Minimum Essential Medium $\alpha$ with $10 \%$ fetal bovine serum (FBS), 402 $1 \times$ GlutaMAX, and $0.15 \%$ sodium bicarbonate in a humidified 403 incubator at $37{ }^{\circ} \mathrm{C}$ and $5 \% \mathrm{CO}_{2}$. Cells were cultured without 404 antibiotics or antimycotics. Cells were washed with PBS, trypsinised, 405 collected by centrifuge $(1000 \mathrm{~g}, 5 \mathrm{~min})$, and subcultured before 406 reaching $80 \%$ confluence. Passage numbers were kept low for all 407 experiments.

IC50 Analysis. Cells were plated in 96 well plates at 5000 cells/ 409 well in $50 \mu \mathrm{L}$ of media. Plates were incubated for $24 \mathrm{~h}$ and checked 410 visually for consistent cell density before treatments were applied. 411 Nanoparticle concentrations were calculated by diluting according to 412 total DTX mass from HPLC loading analysis. DTX and nanoparticle 413 stock solutions were prepared in a minimum of 7 concentrations 414 between 10 and $0.0001 \mathrm{mg} / \mathrm{mL}$ in DMSO. The stocks were sterilized 415 with ultraviolet light, then diluted 1 in 250 parts media. Treatments in 416 media were applied in triplicate at $50 \mu \mathrm{L}$ per well then thoroughly 417 agitated. Treatments containing DMSO were diluted by $1 / 2$ by 418 addition to media in wells, for a total of $0.2 \% \mathrm{v} / \mathrm{v}$ DMSO in $100 \mu \mathrm{L}$ of 419 media per well. The outer wells of each plate were filled with PBS to 420 maintain even evaporation in treated cells. Each plate also contained 421 three wells of cells with $100 \mu \mathrm{L}$ of media and three with $100 \mu \mathrm{L}$ of 422 media $+0.2 \% \mathrm{v} / \mathrm{v}$ DMSO as controls. Treatments were allowed to 423 incubate with cells for a total of $24 \mathrm{~h}$ at which point cell culture media 424 containing each of the treatments were removed by inverting the 425 plate, gently shaking, and dabbing onto a sterile paper towel. Cells 426 were then immediately washed twice with PBS (100 $\mu \mathrm{L}$ per well), 427 agitated for $1 \mathrm{~min}$, then the PBS was removed. Warmed media was 428 immediately applied ( $100 \mu \mathrm{L}$ per well). Plates were then incubated for 429 $48 \mathrm{~h}$, accumulating to a total $72 \mathrm{~h}$ of in vitro study period. $\quad 430$

Assays were performed using warmed CellTiter 96 AQueous One 431 Solution Cell Proliferation Assay ((3-(4,5-dimethylthiazol-2-yl)-5-(3- 432 carboxymethoxyphenyl)-2-(4-sulfophenyl)-2H-tetrazolium), "MTS") 433 solution at $20 \mu \mathrm{L}$ per well. Plates were read $3 \mathrm{~h}$ after the reagent was 434 applied with a PerkinElmer EnSpire Multimode plate reader. 435 Absorbance was measured at $490 \mathrm{~nm}$ in triplicate and averaged. 436 IC50 curves were constructed from triplicate assay data in GraphPad 437 
438 Prism. Data sets were normalized, then analyzed by 4-parameter 439 nonlinear regression with an inhibition model.

440 EGFR Blocking Experiment. MDA-MB-231 cells were plated in 44196 well plates at 5000 cells/well in $50 \mu \mathrm{L}$ of media. After $24 \mathrm{~h}$, cells 442 were treated with a blocking antibody anti-EGFR (H11, Life 443 Technologies, catalog no. MA5-13070) at $1 \mu \mathrm{g} / \mathrm{mL}$ or PBS (control) 444 diluted in media for $1 \mathrm{~h}$ followed by a $24 \mathrm{~h}$ incubation with either 445 targeted GE11-PGMA-DTX-NPs or untargeted PGMA-DTX-NPs at 446 their determined IC50 concentrations with MDA-MB-231 cells. At a 447 time $24 \mathrm{~h}$ after the incubation, media was changed for fresh media and 448 the plate remained in the incubator for a further $48 \mathrm{~h}$. After this time, 449 cell viability was assessed using CellTiter-Glo 2.0 following the 450 manufacturer's instructions.

451 Confocal Imaging. MDA-MB-231 cells were cultured as above 452 on glass coverslips in 6-well plates and treated for $24 \mathrm{~h}$ with 453 nanoparticle formulations $(10 \mu \mathrm{g} / \mathrm{mL})$, before thorough washing with 454 PBS and fixation with $4 \%$ paraformaldehyde. Samples were 455 immunohistochemically labeled with Mouse anti-EGFR Monoclonal 456 antibody (1:1000, 3\% BSA in PBS, $4{ }^{\circ} \mathrm{C}$, overnight), washed with PBS 457 (twice, $5 \mathrm{~min}$ ), and labeled with goat antimouse Alexa Fluor 488 458 (1:500 in Fluorobrite, $37{ }^{\circ} \mathrm{C}, 30 \mathrm{~min}$ ). Secondary was then removed, 459 washed with PBS (twice, $5 \mathrm{~min}$ ), and stained with Hoechst (1:1000, 460 PBS, RT, $20 \mathrm{~min}$ ) before washing with PBS (twice, $5 \mathrm{~min}$ ). 461 Colocalization studies were treated as per the above samples with the 462 following antibodies (endosomes, $1^{\circ}$ rabbit-EEA1, $2^{\circ}$ antirabbit 463 AF488 and lysosomes, $1^{\circ}$ mouse-LAMP1, $2^{\circ}$ antimouse AF647. 464 Samples were mounted on glass microscope slides and imaged on a 465 Nikon Ti-E inverted confocal microscope with a Nikon A1Si spectral 466 detector system and a Nikon $60 \times$ oil immersion, 1.49NA objective. 467 For super resolution imaging, images were captured on the Nikon N468 SIM system with a Nikon SR 100×, 1.49NA objective. Cells were 469 treated as above but were seeded on a coverglass of accurate thickness $470(170 \pm 5 \mu \mathrm{m}$ from Marienfeld, Germany) to aid in reconstruction 471 efficiency.

472 Statistical Analysis. All results are displayed as means \pm SD. Data 473 was analyzed using the GraphPad Prism version 6.0 data management 474 software to conduct ANOVA on groups of data. Statistically 475 significant differences between each treatment were determined 476 using Bonferroni post hoc tests $(* \# p<0.05, * * p<0.01$, ***p $<$ $4770.001, * * * * P<0.0001)$.

\section{ASSOCIATED CONTENT}

\section{S Supporting Information}

480 The Supporting Information is available free of charge on the 481 ACS Publications website at DOI: 10.1021/acs.lang482 muir.8b02946.

483 Complete synthetic procedures and protocols, support484 ing experiments including RP-HPLC standard curve and 485 drug loading for docetaxel, thiol assay quantification, 486 supporting confocal imaging analysis, SIM video links, 487 and Pearson colocalization analysis of nanoparticles with 488 endosomes or lysosomes (PDF)

\section{AUTHOR INFORMATION}

\section{ORCID $\odot$}

491 Tristan D. Clemons: 0000-0001-8042-0141

492 Anabel Sorolla: 0000-0001-8238-8763

493 K. Swaminatha Iyer: 0000-0001-9329-4930

\section{Author Contributions}

${ }_{495}{ }^{\perp}$ T.D.C. and R.S. contributed equally to the manuscript.

496 Notes

497 The authors declare no competing financial interest.

\section{ACKNOWLEDGMENTS}

498

The authors acknowledge the Australian Microscopy \& 499 Microanalysis Research Facility at the Centre for Microscopy, 500 Characterization \& Analysis, The University of Western 501 Australia, funded by the University, State and Commonwealth 502 Governments. This work was funded by the Australian 503 Research Council (ARC), the National Health \& Medical 504 Research Council (NHMRC), and the Raine Medical Research 505 Foundation of Australia. Tristan D. Clemons is a NHMRC 506 Peter Doherty-Australian Biomedical Fellow. Anabel Sorolla 507 is a recipient of a National Breast Cancer Foundation 508 postdoctoral fellowship, and K. Swaminathan Iyer is an ARC 509 Future fellow.

\section{ABBREVIATIONS}

511

BSA, bovine serum albumin; DTX, docetaxel; EGFR, 512 epidermal growth factor receptor; EPR effect, enhanced 513 permeation and retention effect; FDA, United States Food 514 and Drug Administration; GE11, epidermal growth factor 515 targeting peptide; GE11-PGMA-DTX-NPs, polymeric nano- 516 particles loaded with docetaxel and containing the GE11 517 targeting peptide; IC50, half maximal inhibitory concentration; 518 PBS, phosphate buffered saline; PGMA, poly(glycidyl meth- 519 acrylate); PGMA-DTX-NPs, nontargeted polymeric nano- 520 particles loaded with docetaxel; RP-HPLC, reverse phase 521 high-pressure liquid chromatography; SIM, structured illumi- 522 nation microscopy

\section{REFERENCES}

(1) Stewart, B. W., Kleihues, P., Eds. World Cancer Report 2003; 525 IARC Press: Lyon, France, 2003; p 351 p. 526

(2) DeVita, V.; Chu, E. A History of Cancer Chemotherapy. Cancer 527 Res. 2008, 68 (21), 8643-8653.

(3) Love, R. R.; Leventhal, H.; Easterling, D. V.; Nerenz, D. R. Side 529 effects and emotional distress during cancer chemotherapy. Cancer 530 1989, 63 (3), 604-12.

(4) Carey, M. P.; Burish, T. G. Etiology and treatment of the 532 psychological side effects associated with cancer chemotherapy: a 533 critical review and discussion. Psychol. Bull. 1988, 104 (3), 307-25. 534

(5) Brannon-Peppas, L.; Blanchette, J. O. Nanoparticle and targeted 535 systems for cancer therapy. Adv. Drug Delivery Rev. 2012, 64, 206- 536 212.

(6) Cheng, Z. L.; Al Zaki, A.; Hui, J. Z.; Muzykantov, V. R.; 538 Tsourkas, A. Multifunctional nanoparticles: Cost versus benefit of 539 adding targeting and imaging capabilities. Science 2012, 338 (6109), 540 903-910.

541

(7) Farokhzad, O. C.; Cheng, J. J.; Teply, B. A.; Sherifi, I.; Jon, S.; 542 Kantoff, P. W.; Richie, J. P.; Langer, R. Targeted nanoparticle-aptamer 543 bioconjugates for cancer chemotherapy in vivo. Proc. Natl. Acad. Sci. 544 U. S. A. 2006, 103 (16), 6315-6320.

(8) Gu, F. X.; Karnik, R.; Wang, A. Z.; Alexis, F.; Levy-Nissenbaum, 546 E.; Hong, S.; Langer, R. S.; Farokhzad, O. C. Targeted nanoparticles 547 for cancer therapy. Nano Today 2007, 2 (3), 14-21. 548

(9) Shi, J. J.; Kantoff, P. W.; Wooster, R.; Farokhzad, O. C. Cancer 549 nanomedicine: progress, challenges and opportunities. Nat. Rev. 550 Cancer 2017, 17 (1), 20-37.

551

(10) Peer, D.; Karp, J. M.; Hong, S.; FarokHzad, O. C.; Margalit, R.; 552 Langer, R. Nanocarriers as an emerging platform for cancer therapy. 553 Nat. Nanotechnol. 2007, 2 (12), 751-760.

554

(11) Zhou, J.; Patel, T. R.; Fu, M.; Bertram, J. P.; Saltzman, W. M. 555 Octa-functional PLGA nanoparticles for targeted and efficient siRNA 556 delivery to tumors. Biomaterials 2012, 33 (2), 583-591. 557 (12) Li, X.; Zhou, H. Y.; Yang, L.; Du, G. Q.; Pai-Panandiker, A. S.; 558 Huang, X. F.; Yan, B. Enhancement of cell recognition in vitro by 559 dual-ligand cancer targeting gold nanoparticles. Biomaterials 2011, 32560 (10), 2540-2545. 
562 (13) Sawant, R. R.; Torchilin, V. P. Multifunctional nanocarriers and 563 intracellular drug delivery. Curr. Opin. Solid State Mater. Sci. 2012, 16 564 (6), 269-275.

565 (14) Matsumura, Y.; Maeda, H. A New Concept for Macromolecular 566 Therapeutics in Cancer-Chemotherapy - Mechanism of Tumoritropic 567 Accumulation of Proteins and the Antitumor Agent Smancs. Cancer 568 Res. 1986, 46 (12), 6387-6392.

569 (15) U.S. National Library of Medicine. ClinicalTrials.gov, https:// 570 clinicaltrials.gov/ct2/show/NCT01300533?term=BIND-014\&rank=3 571 (accessed 2016).

572 (16) Jain, R. K. Barriers to Drug-Delivery in Solid Tumors. Sci. Am. 5731994,271 (1), 58-65.

574 (17) Cho, K.; Wang, X.; Nie, S.; Chen, Z. G.; Shin, D. M. 575 Therapeutic nanoparticles for drug delivery in cancer. Clin. Cancer 576 Res. 2008, 14 (5), 1310-6.

577 (18) Yang, L. L.; Mao, H.; Wang, Y. A.; Cao, Z. H.; Peng, X. H.; 578 Wang, X. X.; Duan, H. W.; Ni, C. C.; Yuan, Q. G.; Adams, G.; Smith, 579 M. Q.; Wood, W. C.; Gao, X. H.; Nie, S. M. Single Chain Epidermal 580 Growth Factor Receptor Antibody Conjugated Nanoparticles for in 581 vivo Tumor Targeting and Imaging. Small 2009, 5 (2), 235-243.

582 (19) Sanfilippo, J. S.; Miseljic, S.; Yang, A. R.; Doering, D. L.; 583 Shaheen, R. M.; Wittliff, J. L. Quantitative analyses of epidermal 584 growth factor receptors, HER-2/neu oncoprotein and cathepsin D in 585 nonmalignant and malignant uteri. Cancer 1996, 77 (4), 710-716.

586 (20) Su, Y. C.; Burnouf, P. A.; Chuang, K. H.; Chen, B. M.; Cheng, 587 T. L.; Roffler, S. R. Conditional internalization of PEGylated 588 nanomedicines by PEG engagers for triple negative breast cancer 589 therapy. Nat. Commun. 2017, 8, 15507.

590 (21) Herbst, R. S.; Shin, D. M. Monoclonal antibodies to target 591 epidermal growth factor receptor-positive tumors - A new paradigm 592 for cancer therapy. Cancer 2002, 94 (5), 1593-1611.

593 (22) Song, S. X.; Liu, D.; Peng, J. L.; Sun, Y.; Li, Z. H.; Gu, J. R.; Xu, $594 \mathrm{Y}$. H. Peptide ligand-mediated liposome distribution and targeting to 595 EGFR expressing tumor in vivo. Int. J. Pharm. 2008, 363 (1-2), 155596161.

597 (23) Li, Z.; Zhao, R.; Wu, X.; Sun, Y.; Yao, M.; Li, J.; Xu, Y.; Gu, J. 598 Identification and characterization of a novel peptide ligand of 599 epidermal growth factor receptor for targeted delivery of therapeutics. 600 FASEB J. 2005, 19 (14), 1978-85.

601 (24) Shewmake, T.; Solis, F.; Gillies, R.; Caplan, M. Effects of Linker 602 Length and Flexibility on Multivalent Targeting. Biomacromolecules 603 2008, 9, 3057-3064.

604 (25) Liu, Y.; Cai, Y.; Liu, W.; Li, X.; Rhoades, E.; Yan, E. Triblock 605 peptide-linker-lipid molecular design improves potency of peptide 606 ligands targeting family B G protein-coupled receptors. Chem. 607 Commun. 2015, 51, 6157-6160.

608 (26) Subik, K.; Lee, J. F.; Baxter, L.; Strzepek, T.; Costello, D.; 609 Crowley, P.; Xing, L.; Hung, M. C.; Bonfiglio, T.; Hicks, D.; Tang, P. 610 The Expression Patterns of ER, PR, HER2, CK5/6, EGFR, Ki-67 and 611 AR by Immunohistochemical Analysis in Breast Cancer Cell Lines. 612 Breast Cancer: Basic Clin. Res. 2010, 4, 35-41.

613 (27) Charafe-Jauffret, E.; Ginestier, C.; Monville, F.; Finetti, P.; 614 Adelaide, J.; Cervera, N.; Fekairi, S.; Xerri, L.; Jacquemier, J.; 615 Birnbaum, D.; Bertucci, F. Gene expression profiling of breast cell 616 lines identifies potential new basal markers. Oncogene 2006, 25, $6172273-2284$.

618 (28) Manders, E. M. M.; Stap, J.; Brakenhoff, G. J.; Vandriel, R.; 619 Aten, J. A. Dynamics of 3-Dimensional Replication Patterns during 620 the S-Phase, Analyzed by Double Labeling of DNA and Confocal 621 Microscopy. J. Cell Sci. 1992, 103, 857-862.

622 (29) Dunn, K. W.; Kamocka, M. M.; McDonald, J. H. A practical 623 guide to evaluating colocalization in biological microscopy. Am. J. 624 Physiol-Cell Ph 2011, 300 (4), C723-C742.

625 (30) Evans, C. W.; Fitzgerald, M.; Clemons, T. D.; House, M. J.; 626 Padman, B. S.; Shaw, J. A.; Saunders, M.; Harvey, A. R.; Zdyrko, B.; 627 Luzinov, I.; Silva, G. A.; Dunlop, S. A.; Iyer, K. S. Multimodal analysis 628 of PEI-mediated endocytosis of nanoparticles in neural cells. ACS 629 Nano 2011, 5 (11), 8640-8648.
(31) Kazazic, M.; Bertelsen, V.; Pedersen, K. W.; Vuong, T. T.; 630 Grandal, M. V.; Rodland, M. S.; Traub, L. M.; Stang, E.; Madshus, I. 631 H. Epsin 1 is Involved in Recruitment of Ubiquitinated EGF 632 Receptors into Clathrin-Coated Pits. Traffic 2009, 10 (2), 235-245. 633

(32) Madshus, I. H.; Stang, E. Internalization and intracellular 634 sorting of the EGF receptor: a model for understanding the 635 mechanisms of receptor trafficking. J. Cell Sci. 2009, 122 (19), 636 3433-3439.

637

(33) Clemons, T. D.; Viola, H. M.; House, M. J.; Iyer, K. S.; Hool, L. 638 C. Examining Efficacy of "TAT-less" Delivery of a Peptide against the 639 L-Type Calcium Channel in Cardiac Ischemia-Reperfusion Injury. 640 ACS Nano 2013, 7 (3), 2212-2220.

(34) Singh, R.; Norret, M.; House, M. J.; Galabura, Y.; Bradshaw, 642 M.; Ho, D. W.; Woodward, R. C.; St Pierre, T. G.; Luzinov, I.; Smith, 643 N. M.; Lim, L. Y.; Iyer, K. S. Dose-Dependent Therapeutic 644 Distinction between Active and Passive Targeting Revealed Using 645 Transferrin-Coated PGMA Nanoparticles. Small 2016, 12 (3), 351- 646 359. 